\title{
Queue Performance in Presence of Long-Range Dependencies - An Empirical Study
}

\author{
Barbara Strzałka, Mirosław Mazurek, Dominik Strzałka*
}

Department of Distributed Systems, Rzeszow University of Technology, Al. Powstańców Warszawy 2, 35-959 Rzeszów, Poland Email: strzalka.barbara@gmail.com, \{mirekmaz, strzalka\}@prz.edu.pl

\begin{abstract}
The queuing, as an inherent feature of each real system, plays a very important role in teletraffic networks as well. It is known that the queuing system consists of three basic components: the input stream of requests, the service processes and the queue discipline. The optimal performance of system can be achieved only in the case when all components are "matched" together. The teletraffic models that describe the early telephone services (e.g. Poisson and Markov) have been very successful. One of the main reasons for this success with models, which accurately resembles the real-world behavior, has been due to the highly static, low varying nature of early development phase of telephone voice traffic. This means that the whole environment of early telephone networks can be treated as a simple system. The main features of such systems can be described by: thermodynamic quasi-equilibrium, homogenous topologies, random graph theory, short-range dependencies, Poisson distribution, circuit switching, etc. However, the evolution of teletraffic from voice to new data traffic and integrated services inevitably leads to the vastly different statistical characteristics that are much more irregular and variable and as a result undermine the basis for the traditional traffic models. In this paper we show that taking into account the complex systems approach the existence of long-range dependencies (expressed by $1 / f$ noise) influence queue performance. This is done basing on analytical approach with presentation of long-range dependent queue model and also by experimental study of traffic behavior in computer network during daily workload generated by computer user.
\end{abstract}

Keywords Long-Range Dependencies, Hurst Parameter, Powers Spectral Density, Queues, Complex Systems

\section{Introduction}

The correct performance models require accurate traffic models, which can capture the statistical characteristic of actual traffic. If the traffic models do not represent traffic accurately, one may overestimate or underestimate the network performance. The use of the classical queuing models at present stage of development of network traffic can lead to the mistaken performance predictions and an inadequate network design. Notwithstanding, even the most correct models of particular components of system treated separately do not guarantee the correct model of the whole system. Thus one must take into account, not separately but simultaneously, all processes of systems, i.e. the service process and the queue discipline. This inevitably leads to the analysis based on a complex systems approach [17].

In reality, the simple systems don't exist. In the case of real, complex systems, in contradiction to the simple systems, one can indicate the following features of processes: thermodynamic non-equilibrium, heterogeneous topologies, small-worlds phenomenon, long-range dependencies, bursty

* Corresponding author:

strzalka@prz.edu.pl (Dominik Strzałka)

Published online at http://journal.sapub.org/ijis

Copyright $(C 2012$ Scientific \& Academic Publishing. All Rights Reserved and self-similar traffic, scale-free (power law) distributions, packet switching, structure hierarchy, percolation, clustering, self-organization, parameters degradation and collapse.

The statistical characteristic of teletraffic systems has been changed a lot when the human behavior was replaced by the hierarchical, complex system, so-called computer. On the other hand, the voice traffic was quite static and low variable (short-range dependent) but now the data traffic is much more variable with both the extremely short and the extremely long calls (self-similar and long-range dependent). Thus, it can be noticed that both the original "pure form" human behavior and the original "pure" traffic nature (a simple stream) are lost when higher (nested) layers of stack were added successively and the simple computer system becomes a complex system that has a fractal nature. It is well known that the nesting concerns all areas of computer engineering (networks, computer hardware, operating systems, programming language and queuing system as well) and inevitably leads to the long-term dependent processes from the short-range dependent processes. It is particularly intensified in complex large-scale systems, i.e. distributed systems [1]. The positive features of system namely: heterogeneity, openness, security, scalability, failure handling, concurrency and transparency are understated by negative complex system features such as degradation and collapse. 
In the case of queue, its hierarchical, nested, layer structure should also be noticed: from bottom physical layer (hardware) to the upper software (service) layer. This nested structure inevitably leads to the fractal phenomena and long-range dependencies. Due to the lack of the general models of queue, all current models treat the queuing systems as simple systems [16]. These models are only special cases, which are not coherent. The main motivation for considerations presented in this paper is a belief that exists a general analytical solution, based on a power spectral density analysis, of a queuing system treated as a complex system (holistically). In order to confirm this conviction we also show a experimental study related to behavior of queue that shows the number of received bytes by one computer in local area network during one day. Details of this experiment will be given in Section 4.

The paper consists of five sections. In Section 2 the short-range and the long-term dependent input stream models have been described. A Section 3 concerns a new, general model of queue based on $1 / f$ noise. Results of experiment are given in Section 4. The paper is crowned in Section 5 that provides the conclusions.

\section{A Brief Introduction to Short-Range and Long-Range Dependent Models}

There have been many mathematical models for queuing systems as long as the systems have existed themselves. However, the changing structure of traffic, range from analogue telephony through its digital version and finally to integrated networks, requires for the existing models, continuously checking for validity. The short-range dependent models include all Poisson processes and Markov processes.

The first mathematical models used to describe old queues $[2,3]$ were based on a renewal process where requests are independent and identically distributed (i.i.d.). This assumption implies that the arrivals to queue are memory-less and short-range dependent. Thus the autocorrelation function for all lags $k \neq 0$ is equal to zero. According to Poisson process the $n$-th inter-arrival time $A_{n}$ of requests stream can be described by the exponential distribution:

$$
P=\left\{A_{n} \leq \tau\right\}=1-e^{-\lambda \tau}
$$

with the mean number of requests arrival per time unit $\lambda$. The number of arrivals within interval of length $\tau$ is:

$$
P=(N(\tau))=e^{-\lambda \tau} \frac{(\lambda \tau)^{n}}{n !}
$$

However, the current traffic has got vastly different features, which are much more irregular, variable and it undermines the basis for traditional models. The first step to describe the dependencies between successive requests was given by Markov models. A Markov property implies that the future depends on current state and neither on previous states nor on time that has been already spent in a current state. This restricts the random variable, which describes time, to the geometric distribution in a discrete case. The Markov models have the correlation structure that is significant only for relatively small lags. A Markov model has got many modifications such as: the ON-OFF model, the modulated model [4], the flow model [5], [6], etc. All of these models are proper when there is no correlation between requests and arrivals or in case when the correlation of time requests is weak.

First of all the authors would like to consider some basic terms of long-range memory dependencies [7]. It is well known that a random process $X$ is strictly stationary, if its probability distribution doesn't change over time, i.e. it's the same at all time points $t$. The wide-sense stationary means that the first and the second order statistical properties, namely the mean:

$$
\mu=E[X(t)]
$$

and the variance:

$$
\sigma^{2}=E\left[(X(t)-\mu)^{2}\right]
$$

don't change over time.

Given a wide-sense stationary process $X$, the definition of the autocovariance function of $X$ is:

$$
R(\tau)=E[(X(t)-\mu)(X(t-\tau)-\mu)]
$$

and the autocorrelation function of $X$ :

$$
\rho(\tau)=\frac{R(\tau)}{R(0)}=\frac{R(\tau)}{\sigma^{2}}
$$

Taking into account a wide-sense stationary random process, it can be particularly assumed that its autocorrelation function is expressed by:

$$
\rho(\tau) \rightarrow \tau^{-\beta} L(\tau) \text { for } \tau \rightarrow \infty
$$

where $0<\beta<1$ and $L(\tau)$ is slowly varying at infinity, that is $\lim _{\tau \rightarrow \infty} \frac{L(\tau x)}{L(\tau)}=1$, for all $x>0$.

Let $X^{(m)}$ denote the new process obtained by averaging the original series $X$ into non-overlapping sub-blocks of size $m$. That is:

$$
X^{(m)}(t)=\frac{X_{t m-m+1}+X_{t m-m+2}+\ldots+X_{t m}}{m}
$$

Note that for each $m, X^{(m)}$ defines a wide-sense stationary process. Let $\rho^{(m)}(\tau)$ be the autocorrelation function of $X^{(m)}$. One can say that the process $X$ is exactly second-order self-similar with Hurst parameter $H=1-\beta / 2$, if the aggregated processes have the same autocorrelation structure as $X$. That is:

$$
\rho^{(m)}(\tau)=\rho(\tau) \text { for all } m=1,2, \ldots
$$

In other words, $X$ is exactly second-order self-similar if the aggregated processes $X^{(m)}$ are indistinguishable from $X$ with respect to their first and second order properties. One can relax this requirements and say that $X$ is asymptotically second-order self-similar if:

$$
\rho^{(m)}(\tau) \approx m^{1-\beta} \quad \text { when } \quad m \rightarrow \infty
$$


The most interesting feature of statistical self-similarity is that the correlation of aggregated processes doesn't degenerate as $m \rightarrow \infty$. This is in contradiction to the traditional models, which all have the property that the correlation structure of their aggregated processes degenerates as $m \rightarrow \infty$, i.e.:

$$
\rho^{(m)}(\tau) \rightarrow 0 \text { when } m \rightarrow \infty \text { for all } m=1,2, \ldots
$$

A process satisfying (7) is said to be the long-range dependent. This means that such processes can be characterized by the autocorrelation function, which decays hyperbolically as $\tau$ increases. The short-range dependent processes have got autocorrelation function that decays exponentially as $\tau$ increases.

All contemporary LRD and self-similarity traffic models are based on the fractional Brownian motion ( $\mathrm{fBm})$, which is a generalization of the more known Brownian motion process. It is a centered Gaussian process with stationary increments (the fractional Gaussian noise - fGn). However, the increments of $\mathrm{fBm}$ aren't independent, except the standard Brownian motion. The dependence structure of increments is modeled by the Hurst parameter $H \in\langle 0,1\rangle$.

Kolmogorov originally introduced the $\mathrm{fBm}$ in [8]. The name fBm comes from Mandelbrott and Van Ness [9].

The fractional Gaussian noise is the stationary Gaussian process with mean $\mu$, variance $\sigma^{2}$ and autocorrelation function:

$$
\rho(\tau)=\frac{|\tau+1|^{2 H}+|\tau|^{2 H}+|\tau-1|^{2 H}}{2}
$$

It can be easily showed that:

$$
\rho(\tau) \sim H(2 H-1)|\tau|^{2 H-2} \text { for } \tau \rightarrow \infty
$$

Simultaneously the aggregated processes $X^{(m)}$ all have the same distribution as $X$ for all $0<H<1$. Thus, by (7) and (9), fGn is exactly self-similar with the Hurst parameter $H$ as long as $0<H<1$, and has got the long memory property when $H>0.5$.

The spectral density function of fGn is given by [10]:

$$
S(f)=4 \sigma^{2} C_{H} \sin ^{2}(\pi f) \sum_{j=-\infty}^{\infty} \frac{1}{|f+j|^{2 H+1}}|f| \leq \frac{1}{2}
$$

where $\sigma^{2}$ is the variance of process and:

$$
C_{H}=\Gamma(2 H+1) \frac{\sin (\pi H)}{2 \pi^{2 H+1}}
$$

The equation (14) can be written in a different form:

$$
S(f) \propto f^{-\beta}
$$

where $\beta=2 H-1$. Thus the condition $0.5<H<1$ leads to the excess, long-range dependent $1 / f$ noise. In the case, when $H=1$ one has got a some kind of threshold between the persistent-stationary noise $(0.5<H<1$ or $0<\beta<1)$ and the non-stationary noise (when $\beta>1$, then $\beta=2 H+1$ ). Note that the common term "random" or "white noise" is characterized by Poisson and Markov processes $(H=0.5$ or $\beta=0)$, whereas the random walk or Brownian motion is characterized by $\beta=2$ $(H=0.5)$. The noise characterized by $0<H<0.5$ is called anti-persistent, whereas the noise characterize by $0.5<H<1$ is called persistent.

\section{Long-range Dependent Queue Model}

In the last two decades in computer science, biophysics, economy, etc. we have been the witness of explosion of phenomena identification having the long-term dependencies and the probability densities that extend far beyond the typical tail region of Gaussian distributions. These processes have been classified as the $1 / f$ phenomena; since their series have the power-law spectrum or their probabilities are governed by the power-law.

Instead of analyzing the stochastic processes in the time domain as it is in the classical queuing systems, the processes can also be analyzed in the spectral domain [13]. In this section the authors focus on only the stationary processes. The fundamental assumption of the proposed model is: $1 / f$ noise is the disturbance measure of queue traffic. The existence of direct relationship between the long-range dependent (LRD) processes and the energy of excess $1 / f$ noise is assumed. LRD lead to the queue traffic disturbances and the waiting time of queue. On the other hand, the short-range dependent (SRD) processes is related to the energy of white noise and the service time of requests. These considerations lead to the analytical dependencies between the response time and the service time at Hurst parameter $H$, the bandwidth $W$ and the borderline value of frequency $f_{g}$ between the SRD and LRD processes.

Taking into account (Fig.1), one can notice that in the case of lack of traffic disturbances the queuing system can be viewed as a system where the energy dissipation by queuing is only connected with the short-range dependent processes (white noise) and expressed, instead of analysis of stochastic processes in the time domain, by [10]:

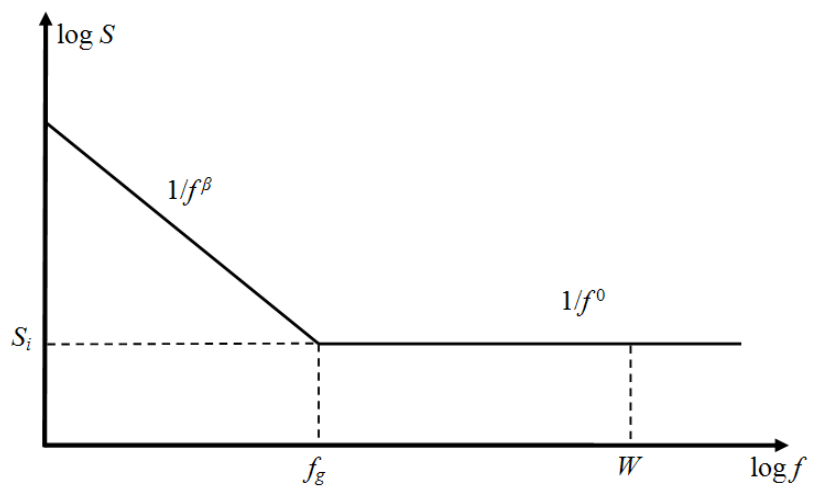

Figure 1. The relationships between the white noise and the excess $1 / f$ noise

$$
\bar{E}_{i}=S_{i} \int_{0}^{W} \frac{1}{f^{0}} d f \cdot \bar{t}_{i}=S_{i} W \overline{t_{i}}
$$

Where $S_{i}$ is the power spectral density of white noise, $W$ is the bandwidth of queuing system, $f_{g}$ is the borderline frequency, $\bar{t}_{i}$ is the mean value of response time in a non-disturbanced (ideal) system. In the case when traffic is disturbanced, besides the short-range dependent processes and white noise, the long-range dependent processes with $1 / f$ noise appear. Then the energy dissipation in such a system is connected to both the short-range and the long-range dependent processes: 


$$
\bar{E}_{r}=S_{i}\left(\int_{f_{g}}^{W} \frac{1}{f^{0}} d f+\int_{0}^{f_{g}} \frac{1}{f^{\beta}} d f\right) \bar{t}_{r}=S_{i}\left(W-f_{g}+\frac{f_{g}^{2-2 H}}{2-2 H}\right) \bar{t}_{r}
$$

where $\bar{t}_{r}$ is the mean value of response time in disturbanced, real system, $f_{g}$ is the borderline value of frequency between the SRD and LRD processes.

The comparison of two cases leads to a general solution of (complex) queuing system:

$$
\frac{S_{i}\left(W-f_{g}+\frac{f_{g}^{2-2 H}}{2-2 H}\right)}{\bar{t}_{r}}=\frac{S_{i} T}{\bar{t}_{i}}
$$

and finally:

$$
\bar{t}_{r}=\bar{t}_{i} \frac{W-f_{g}+\frac{f_{g}^{2-2 H}}{2-2 H}}{W}
$$

Taking into account Eq. (20) one can determine two basic characteristics of queuing system, i.e. $t_{r} / t_{i}=f(W)$ at $H=p a r$ and $f_{g}=\operatorname{par}\left(\right.$ Fig.2) and $t_{r} / t_{i}=f(H)$ at $W=$ par and $f_{g}=\operatorname{par}($ Fig.3).

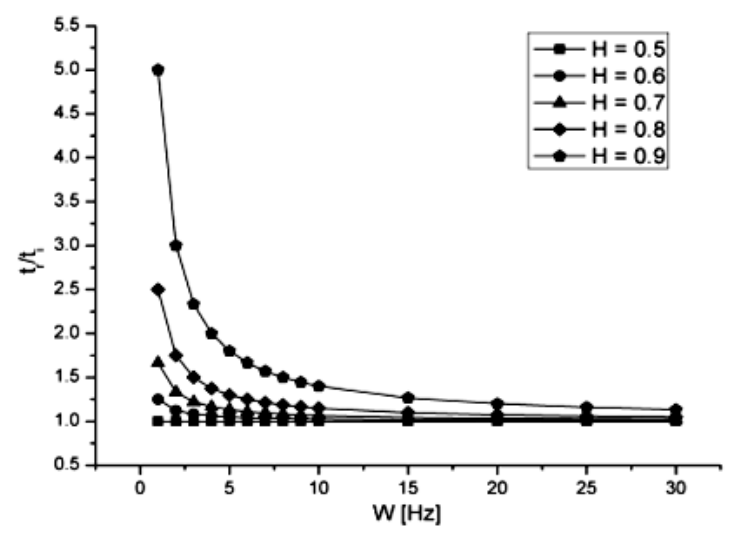

Figure 2. Characteristic of queuing system $t_{r} / t_{i}=f(W)$ at different $H=p a r$ and $f_{g}=1 \mathrm{~Hz}$

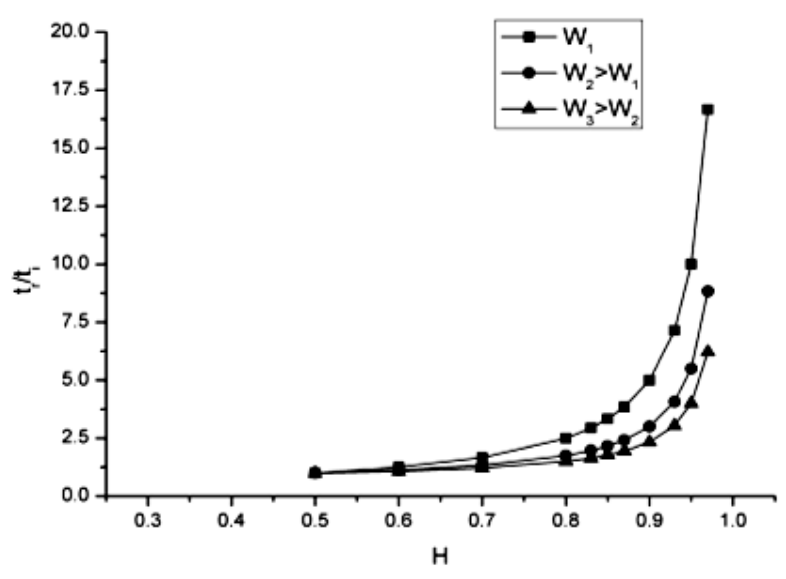

Figure 3. Characteristic of queuing system $t_{r} / t_{i}=f(H)$ at different $W=p a r$ and $f_{g}=1 \mathrm{~Hz}$

From (Fig.2) one can see that $t_{r} / t_{i}$ increases rapidly when the self-similarity parameter $H$ raises and the deep long memory dependencies exist if $W$ is small. This means that if one wants to guarantee $t_{r}$ close to $t_{i}$ then will need a bigger $W$. This observation is confirmed on (Fig.3) where it is clearly visible that bigger $W$ guarantees that $t_{r} / t_{i}$ will be acceptably small.

\section{Analysis}

In this Section we show the results of experiment. It was carried on one computer that was connected to computer network. Data were collected during one day (24h) with interval $1 \mathrm{~s}$ thus we collected above $86 \mathrm{k}$ of observations. Collection was obtained thanks to the perfmon tool that is a an administration program under Windows operating systems. It allows for tracing of many different counters (especially queues) in computer system. Fig. 5 shows behavior of traced counter, i.e., received traffic from network during normal work of computer user. The term 'normal work' should be considered as a situation where there aren't performed any special benchmarks in order to obtain data collected during experiment - we consider only such a traffic that comes from user behavior during tracing. As one can see (Fig. 4) there are intervals of high workload and also such with low traffic. We started data collection on 9:30 and this is well visible in Fig. 4 - during night hours traffic is very low. Considered user didn't generate high workload but his computer was turned-on and used in computer network.

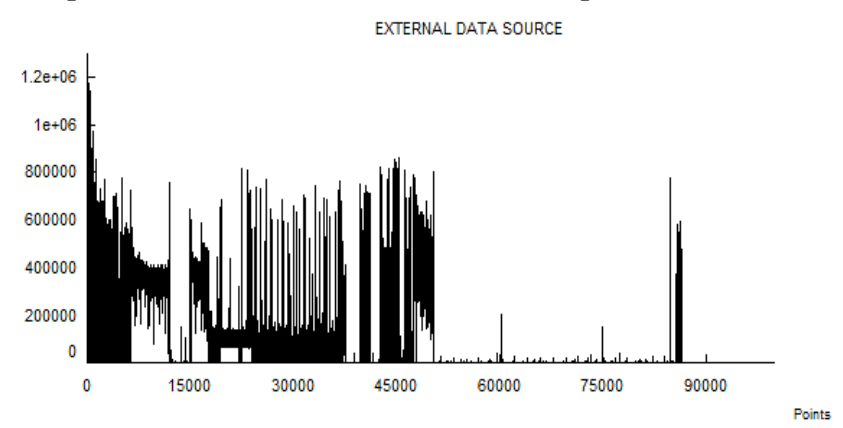

Figure 4. Output traffic in bytes/s during $24 \mathrm{~h}$

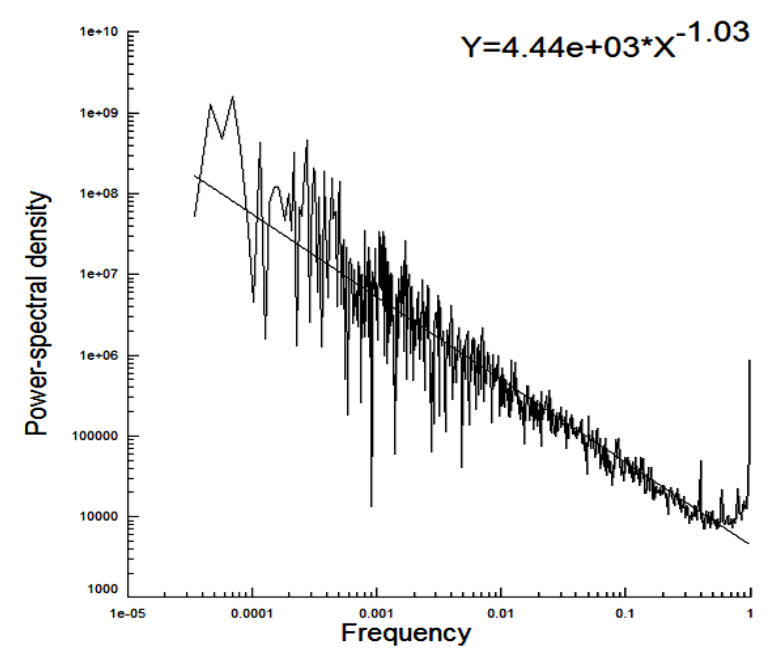

Figure 5. Power-spectral density of output flow for 24 hours of observation

In order to calculate the possible existence of long-range dependencies that can govern collected data and influence the behavior of queue as it was shown above especially in Fig. 2 and Fig. 3 we used power spectral density method. Usually it is considered that this method calculates the degree of long-range dependencies, regardless of whether a process belongs to Gaussian or power-law probability 
distributions domains of attraction. Obviously, the choice for this method is also connected with theoretical considerations presented in Sect. 3. Fig. 5 shows one example of this method usage and taking into account consideration presented in Sect. 2 we have an example of pure 1/f noise. Usually it is hard to calculate a real value of spectral density slope because of the usage of last mean square method that not necessary is good for log-log plots, but it is commonly accepted that such an approach can be taken for rough estimation of $H$ parameter.

In Table 1 one can find results of analysis obtained from
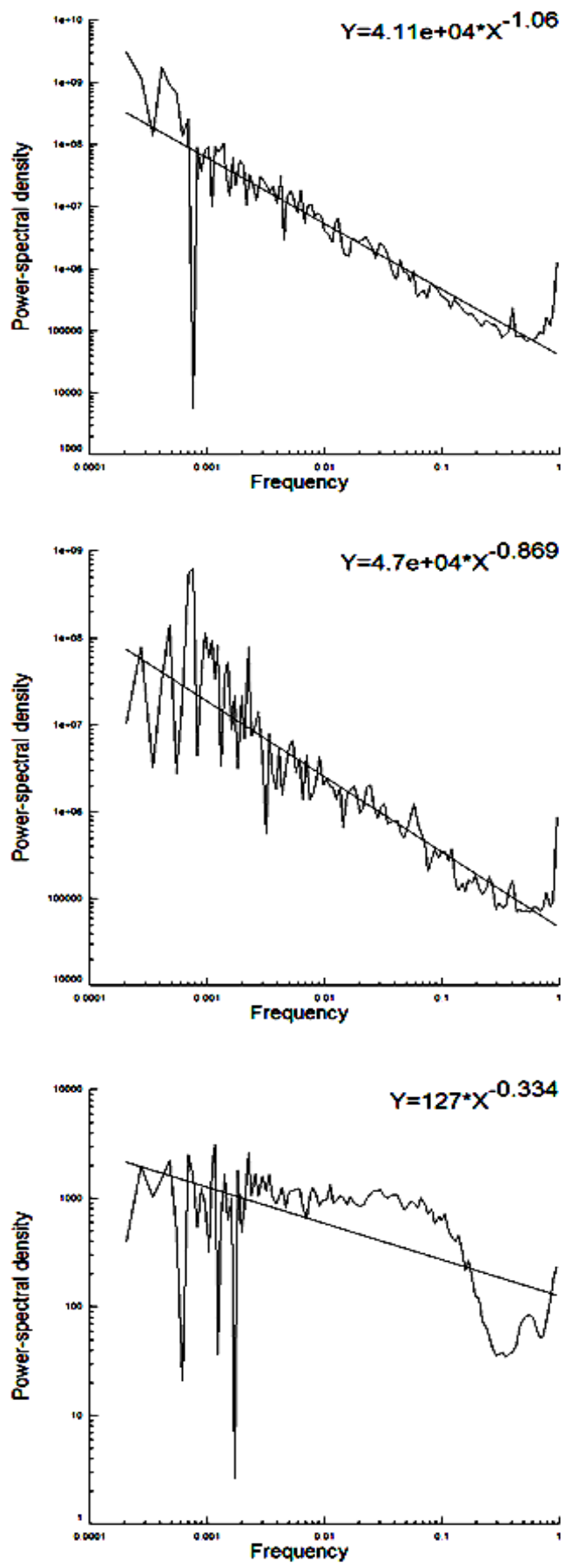

Fig. 6. Because we didn't check the probability distribution of considered process, it cannot be told that the slope of spectral density represents self-similarity parameter $H$, but it for sure shows the degree of long range dependencies $d$ (see details for example in [11]). We decided to divide our collection on 24 subcollections (they represent traffic during 1 hour) and calculate for them the slope of spectral density (results in Table 1, columns $\beta_{h}$ and $d_{h}$ ) and also we took the overlapping $4 \mathrm{~h}$ periods in two different ways (columns $\beta^{\prime}{ }_{4 h}$, $d^{\prime}{ }_{4 h}, \beta{ }^{\prime}{ }_{4 h}, d^{\prime \prime}{ }_{4 h}$ ). For $\beta>0$ there is no possibility to calculate $d$.
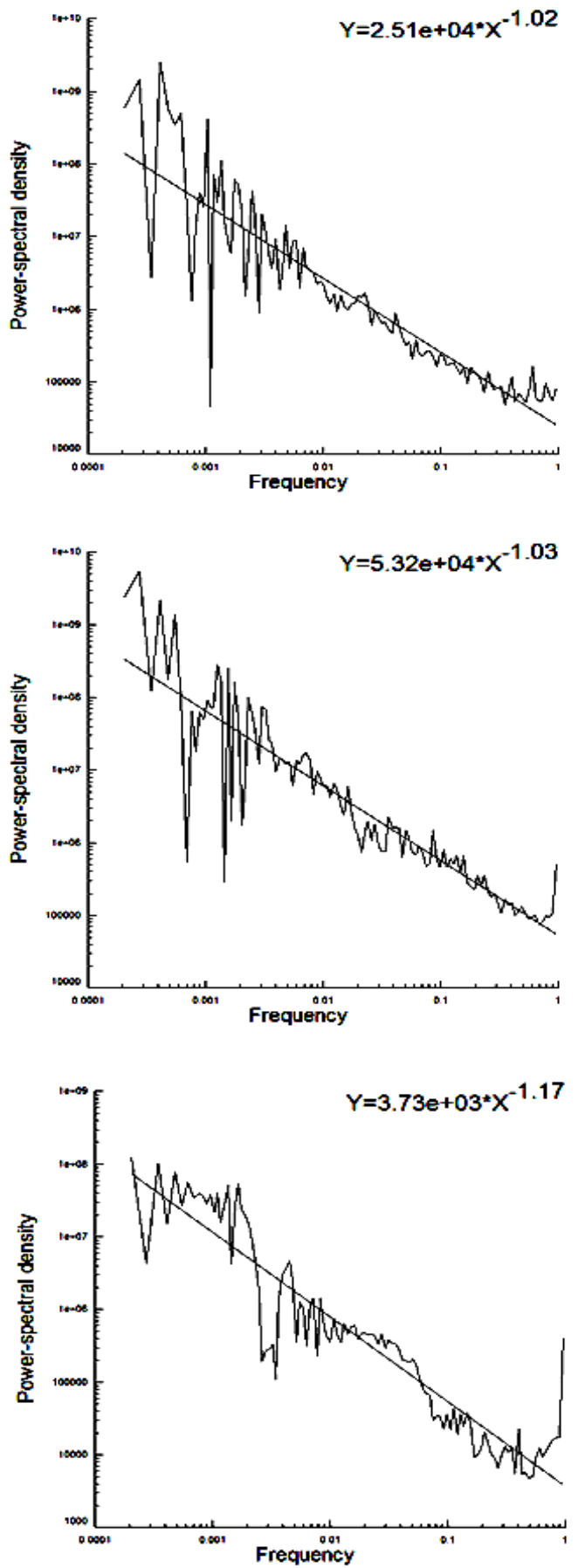

Figure 6. Power spectral density for different $4 \mathrm{~h}$ periods of analyzed traffic 
Table 1. Results of power spectral density analysis for different subsets of collected data

\begin{tabular}{|c|c|c|c|c|c|c|}
\hline Hour & $\beta_{h}$ & $d_{h}$ & $\beta_{4 h}^{\prime}$ & $d_{4 h}^{\prime}$ & $\beta^{\prime,}{ }_{4 h}$ & $d^{\prime \prime}{ }_{4 h}$ \\
\hline 1 & -0.765 & 0.8825 & \multirow{4}{*}{-1.06} & \multirow{4}{*}{$0.03 *$} & \multirow{2}{*}{-0.933} & \multirow{2}{*}{0.9665} \\
\hline 2 & -1.04 & $0.02 *$ & & & & \\
\hline 3 & -0.386 & 0.693 & & & \multirow{4}{*}{-1.36} & \multirow{4}{*}{0.18} \\
\hline 4 & -1.7 & 0.35 & & & & \\
\hline 5 & -1.39 & 0.195 & \multirow{4}{*}{-1.02} & \multirow{4}{*}{$0.01 *$} & & \\
\hline 6 & -0.772 & 0.886 & & & & \\
\hline 7 & -0.641 & 0.8205 & & & \multirow{4}{*}{-0.619} & \multirow{4}{*}{0.8095} \\
\hline 8 & 0.256 & $\mathrm{xxx}$ & & & & \\
\hline 9 & -0.206 & 0.603 & \multirow{4}{*}{-0.869} & \multirow{4}{*}{0.9345} & & \\
\hline 10 & -0.17 & 0.585 & & & & \\
\hline 11 & -0.966 & 0.983 & & & \multirow{4}{*}{-0.942} & \multirow{4}{*}{0.971} \\
\hline 12 & -1.12 & $0.06^{*}$ & & & & \\
\hline 13 & -0.779 & 0.8895 & \multirow{4}{*}{-1.03} & \multirow{4}{*}{$0.015^{*}$} & & \\
\hline 14 & -1.16 & $0.08 *$ & & & & \\
\hline 15 & -1.38 & 0.19 & & & \multirow{4}{*}{-1.09} & \multirow{4}{*}{$0.045^{*}$} \\
\hline 16 & 0.171 & $\mathrm{xxx}$ & & & & \\
\hline 17 & -0.19 & 0.595 & \multirow{4}{*}{-0.334} & \multirow{4}{*}{0.667} & & \\
\hline 18 & 0.335 & $\mathrm{xxx}$ & & & & \\
\hline 19 & 0.304 & $\mathrm{xxx}$ & & & \multirow{4}{*}{-0.316} & \multirow{4}{*}{0.658} \\
\hline 20 & 0.0568 & $\mathrm{xxx}$ & & & & \\
\hline 21 & -0.443 & 0.7215 & \multirow{4}{*}{-1.17} & \multirow{4}{*}{$0.085^{*}$} & & \\
\hline 22 & 0.244 & $\mathrm{xxx}$ & & & & \\
\hline 23 & 0.0896 & $\mathrm{xxx}$ & & & \multirow{2}{*}{-1.19} & \multirow{2}{*}{0.095} \\
\hline 24 & -1.2 & $0.1^{*}$ & & & & \\
\hline day & -1.03 & $0.015^{*}$ & & & & \\
\hline
\end{tabular}

*- because $\beta$ is close to - 1 calculations of $d$ can be given into two different ways: $d=\frac{-\beta+1}{2}$ or $d=\frac{-\beta-1}{2}$

The obtained results of this experiment show that in contradiction to so far existing belief [14] the traffic generated by only one computer can have a self-similar nature. As it can be seen (Table 1) such a property is well visible not only for long periods of time [15] but also for short ones. Power spectral density (as it was shown in Sect. 3) can be used not only for proposal of analytical model (which is also directly related to terms used in statistical mechanics because we have in Eq. (17) energy dissipation) but also to confirm the validity of proposed model basing on simple experiment. In considered case we don't have a workload that was generated by high throughput server or by any special benchmark (test) - but we show that the behaviour of one computer user can lead to traffic self-similar nature.

\section{Conclusions}

The presented paper deals with an analytical, general model of queuing system where the impact of long-range dependent processes on queue performance can be investigated. As a starting point of investigations the assumption that the queues are complex systems in contradiction to the traditional models was assumed. The given assumptions (the self-similar nature of traffic expressed by the fractional Brownian motion $(\mathrm{fBm})$ or the fractional Gaussian noise (fGn) and the holistic approach to queue analysis) made the possibilities to determine the power spectral density $S(f)$ which can be an internal level measure of high variable traffic in the whole system. The direct relationship between the long-range dependent (LRD) processes and the energy of excess $1 / f$ noise was assumed. As it turned out, the LRD led to the queue traffic disturbances and the waiting time of queue. On the other hand the short-range dependent (SRD) processes were related to the energy of white noise and the service time of requests. These considerations led to the analytical dependencies between the mean value of the system response time $t_{r}$ and the mean value of the system service time at Hurst parameter $H$, the bandwidth $W$ and the borderline value of frequency $f_{g}$ between the SRD and LRD processes. Results of analytical considerations were related to simple experiment that showed the properties of computer network traffic. As it turned-out it is also governed by LRD dependencies even when one computer is traced only during $24 \mathrm{~h}$.

\section{REFERENCES}

[1] G. Coulouris, J. Dollimore, T. Kindberg: Distributed Systems. Concepts and Design. Addison-Wesley, (2001).

[2] D. G. Kendall: Some problems in the theory of queues. Journal of the Royal Statistical Society, Ser. B, vol. 13, pp.151-185, (1951).

[3] L. Kleinrock: Queuing systems, vol. 1. John Wiley, New York, (1975).

[4] H. Heffes, D. Lucantoni: A Markov modulated characterization of packetized voice and datatraffic and related statistical multiplexer performance. IEEE JSAC, pp. 856-868, (1986).

[5] H. Hlavacs, G. Kotsis, C. Steinkellner: Traffic source modeling. Tech. Rep. No. TR-99101, Univ. of Vienna, p. 12, (1999).

[6] A. Adas: Traffic models in broadband networks. IEEE Communication Magazine, pp. 82-89, (1997).

[7] W. E. Leland, M. S. Taqqu, W. Willinger, D. V. Wilson: On the self-similar of Ethernet traffic, IEEE/ACM Transactions on Networking, 2(1), pp. 1-15, (1994).

[8] N. Kolmogorov: Wienersche Spiralen und einige andere interessante Kurven im Hilbertschen Raum. Acad. Sci. USSR. 28, 115-118, (1940).

[9] B. Mandelbrot, J. W. van Ness: Fractional Brownian motion, fractional noises and applications. SIAM Review, v. 10, No. 4, pp. 422-437, (1968). 
[10] I. Norros: On the use of fractional Brownian motion in the theory of connectionless networks. IEEE J. Select. Areas Commun., vol. 3, pp. 953-962, (1995).

[11] F. Grabowski, D. Strzałka.: Influence of long-term processes on queue performance. High-performance computer networks - new technologies, pp. 123-133, WNT, Gliwice, (2005) (in Polish).

[12] M. S. Taqqu, V. Teverovsky, W. Willinger: Estimators for Long-Range Dependence: An Empirical Study, Fractals, vol. 3, pp. 785-798, (1995).

[13] S.M. Aqil Burney, A. R. Syed and A. Saleemi: Fast Clustering of Self-Similar Network Traffic Using Wavelet, International Journal of Computer Science Issues, Vol. 7, Issue 3, No 10, p.10, (2010)
[14] O. Sheluhin, S. Smolskiy and A. Osin: Self-Similar Processes in Telecommunications, John Wiley \& Sons, (2007).

[15] M. Fras, J. Mohorko and Ž. Čučej: Modeling of measured self-similar network traffic in OPNET simulation tool, Inf. MIDEM, 40(3), pp. 224-231, (2010).

[16] T. Nakashima: Queue Length Behavior on Restricted Link Under Bursty Self-similar TCP Traffic in Advanced Information Networking and Applications Workshops, WAINA '09. Proc. Of International Conference, pp.: 452 457, (2009)

[17] C.A. Hooker: Philosophy of Complex Systems, Elsevier, (2011) 